\title{
Improvements to ATLAS track reconstruction for Run-2
}

\author{
Hideyuki Oide*, on behalf of the ATLAS Collaboration \\ CERN \\ E-mail: Hideyuki.Oide@cern.ch
}

Run-2 of the LHC will provide new challenges to track and vertex reconstruction with higher energies, denser jets and higher rates. In addition, the Insertable B-layer (IBL) is a fourth pixel layer, which has been inserted at the centre of ATLAS during the shutdown of the LHC. Improvements to track reconstruction developed during the two year shutdown of the LHC are discussed. These include novel techniques developed to improve the performance in the dense cores of jets, optimisation for the expected conditions, and a big software campaign which lead to a factor of four decrease in the CPU time needed to process each recorded event. The commissioning of the detector in preparation for Run-2 using cosmic data and collision data are presented.

The European Physical Society Conference on High Energy Physics

22-29 July 2015

Vienna, Austria

${ }^{*}$ Speaker. 


\section{Introduction}

The ATLAS experiment [1] has successfully taken approximately $25 \mathrm{fb}^{-1}$ of data at $\sqrt{s}=$ $7 \mathrm{TeV}$ and $8 \mathrm{TeV}$ with a bunch crossing space of $50 \mathrm{~ns}$ through the Run-1 of the Large Hadron Collider (LHC). After the long shutdown in 2013-2014 (LS-1), Run-2 started data taking in June 2015 at $\sqrt{s}=13 \mathrm{TeV}$. During Run-2 the LHC is expected to achieve the designed luminosity of approximately $1 \times 10^{34} \mathrm{~cm}^{-2} \mathrm{~s}^{-1}$ with $25 \mathrm{~ns}$ bunch crossing space, and the ATLAS experiment is expected to collect about $100 \mathrm{fb}^{-1}$ of data.

The ATLAS detector is a general-purpose particle detector at the LHC. The Inner Detector measures the momenta and charge of particles through the detector layers by reconstructing their trajectories (tracking) in pseudorapidity range of $|\eta|<2.5^{1}$. It comprises three different subsystems of the Pixel detector (Pixel), silicon strip tracker (SCT), and transition radiation drift tubes (TRT). For Run-2, the Pixel detector was expanded by inserting a new, innermost layer called Insertable B-Layer (IBL) [2]. The IBL has a mean radius of $33.2 \mathrm{~mm}$ and a typical IBL pixel has a size of $50 \mu \mathrm{m}(250 \mu \mathrm{m})$ in the transverse (longitudinal) direction. By adding the IBL the track reconstruction performance is significantly improved as well as, in particular, the impact parameter resolution.

Together with the detector upgrade, the track reconstruction algorithms were overhauled during LS1 and they are much improved compared to Run-1. The overview of these improvements is reported and discussed in Section 2. The early tracking performances using the Run-2 commissioning and collision data are presented in Section 3.

\section{Improvements to track reconstruction}

Software development [3] For Run-2, the event reconstruction throughput at the ATLAS Tier- $0^{2}$ is targeted at $1 \mathrm{kHz}$. To meet this target, the reconstruction speed Inner Detector's track reconstruction, which was taking more than $70 \%$ of the ATLAS total reconstruction time in Run-1, was required to be reduced by a factor 3 compared to the speed of Run-1. Several improvements were implemented in order to achieve this: (i) changing the tracking seeding strategy with taking into account of the IBL hits, (ii) changeover of the linear algebra solver from CLHEP [4] to EIGEN [5], (iii) migration of the FORTRAN-based magnetic field description into $\mathrm{C}++$, and optimization of the track extrapolation codes, (iv) employing template class design in the reconstruction software, (v) re-structuring of the code to reduce inheritance, and (vi) adapting to the new, simplified event data model called xAOD [6]. With these improvements the inner tracking reconstruction speed of the Run-2 software release 20.1 was more than four times faster than the Run-1 software release 17.2 as shown in Figure 1. The full detector reconstruction speed was also improved to be four times

\footnotetext{
${ }^{1}$ ATLAS uses a right-handed coordinate system with its origin at the nominal interaction point (IP) in the center of the detector and the $z$-axis along the beam pipe. The $x$-axis points from the IP to the center of the LHC ring, and the $y$-axis points upwards. Cylindrical coordinates $(R, \phi)$ are used in the transverse plane, $\phi$ being the azimuthal angle around the $z$-axis. The pseudorapidty is defined in terms of the polar angle $\theta$ as $\eta=-\ln \tan (\theta / 2)$. Angular distance is measured in units of $\Delta R \equiv \sqrt{(\Delta \eta)^{2}+(\Delta \phi)^{2}}$.

${ }^{2}$ Tier- 0 is the CERN data center of the Worldwide LHC Computing Grid, and it is responsible for performing the first pass at reconstructing the raw data. It also distributes the raw data and the reconstructed output to Tier- $1 \mathrm{~s}$ (downstream data centers), and reprocesses data when the LHC is not running.
} 
faster. Some additional improvements came technical infrastructure, e.g. switching from 32 bit to 64bit architecture or changing to Scientific Linux 6.

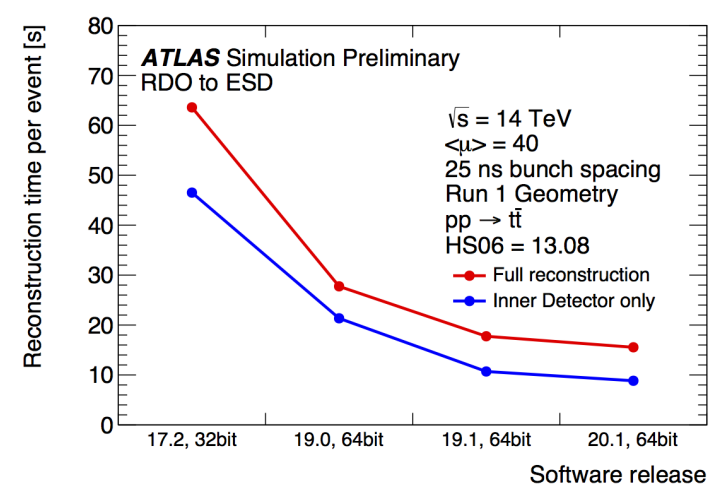

Figure 1: The total reconstruction time per event for a top Monte Carlo simulation sample with 40 pileup at $13 \mathrm{TeV}, 25 \mathrm{~ns}$ bunch spacing. An overall improvement of a factor 4 is visible from the 2012 Tier- 0 release (17.2.7.9), to the Run-2 MC production release 20.1 (64 bit) [3].

Improvement to the performance: tracking in dense environments [7] In Run-1, an artificial neural network (NN) was introduced for Pixel clusters [8] to identify if a cluster is created by a single charged particle, or by multiple charged particles which are sufficiently close so that the clusters are merged using the charge and the topology of the cluster. In case of the Run-1 algorithm, the decision to allow a cluster to be shared by multiple tracks was made before finding track seeds. Identifying merged clusters correctly helps to improve the cluster assignment and track reconstruction efficiency in dense environments as well as decreases the rate at which clusters are used on multiple tracks incorrectly. For Run-2, the decision is delayed downstream of the reconstruction algorithm at the part called the ambiguity solver, where the track candidates are scored for penalization to reduce the number of clusters shared between tracks in highly energetic jets by up to a factor of three. The NN is only consulted when multiple track candidates are sharing the same cluster. If a cluster is judged as to be preferred to be shared by multiple tracks (labelled as shareable), the ambiguity solver doesn't penalize the track candidates which share that cluster. If a cluster is not judged as shareable, the track candidates are penalized when it is shared by multiple track candidates.

With such changes, the tracking efficiency is significantly improved especially for dense environments where multiple tracks traverse narrow areas of the detector layers. Figure 2 demonstrates this improvement for controlled MC particles of two-body decays $\left(\rho \rightarrow \pi^{+} \pi^{-}\right)$and threeprong decays $\left(\tau^{ \pm} \rightarrow \pi^{+} \pi^{-} \pi^{ \pm} v_{\tau}\right.$ ). The algorithmic reconstructed efficiency for $\rho$-daughters and $\tau$-daughters is keeping significantly higher in the Run-2 algorithm in particular at high $p_{\mathrm{T}}$ region where mother particles are largely boosted. The merit of this improvement in more realistic environments is illustrated in Figure 3, where the track reconstruction efficiency is significantly improved in jets and $b$-hadron jets. B-jet efficiency (Figure 3 Right) is calculated at the baseline $70 \%$ working point of the IP3D algorithm [9] as a function of truth-jet transverse momentum. The 
truth jets are reconstructed from generator-level particles in $Z^{\prime}$ events, using the anti- $k_{t}$ algorithm [10] with $R=0.4$, and are required to be within $|\eta|<2.5$.
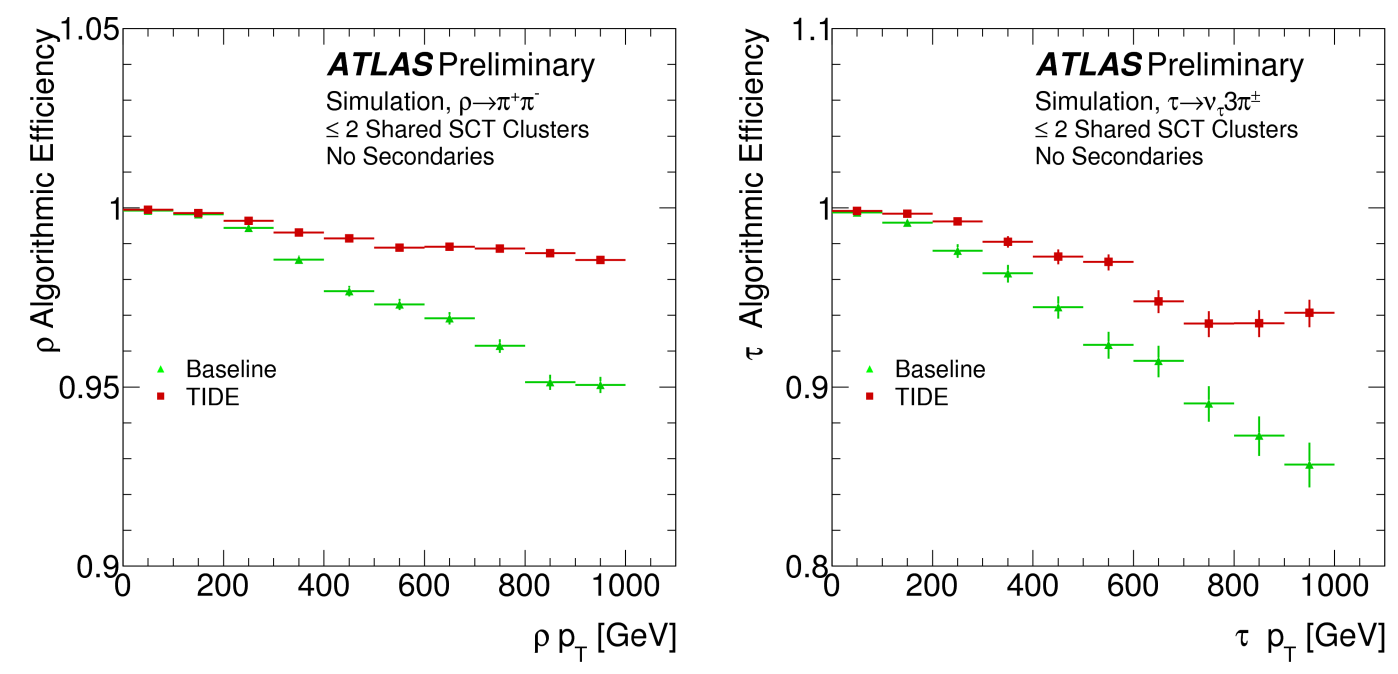

Figure 2: The efficiency to reconstruct all decay products of a $\rho$ (left) or 3-prong $\tau$ (right) in events where truth-based tracks do not share more than two clusters in the SCT is shown as a function of the parent truth particle $p_{\mathrm{T}}$. Events are restricted not to contain any secondary particles through nuclear interactions between the decay products and the detector material. The Baseline legend represents the Run-1 algorithm, and the TIDE (Tracking In Dense Environment) legend represents the Run-2 algorithm [7].

\section{Tracking performance results with the commissioning and early Run-2 data}

Figure 4 (Left) [11] shows the distribution of number of Pixel+IBL hits per reconstructed track. The Loose track selection ( $p_{\mathrm{T}}>400 \mathrm{MeV},|\eta|<2.5$, number of silicon hits $\geq 7$, number of shared ${ }^{3}$ modules $\leq 1$, number of silicon holes ${ }^{4} \leq 2$, number of pixel holes $\leq 1$ ) is applied. Most tracks have 4 hits illustrating the addition of the information from the IBL. The data is compared with a full detector simulation. Figure 4 (Right) [12] shows the transverse impact parameter resolution as a function of $p_{\mathrm{T}}$ for an early special runs of Run-2 where number of collisions per bunch crossing is 0.005 , and the Run- 1 data. The impact parameter resolution is significantly improved especially for low $p_{\mathrm{T}}$ tracks, thanks to the insertion of the IBL closer to the interaction point compared to the existing Pixel detector and the reduction of the material amount from the interaction point to the first measurement point at the IBL.

The alignment of the Inner Detector components is crucial for obtaining good track reconstruction performance. Correction of each module position has to be made well within the impact

\footnotetext{
${ }^{3} \mathrm{~A}$ shared module, is either a hit in the pixel detector which is used by more than one track, or in the case of the SCT two shared hits in the same SCT layer.

${ }^{4}$ Holes are defined as intersections of the reconstructed track trajectory with a sensitive detector element that do not result in a hit. These are estimated by following closely the track trajectory and comparing the hits-on-track with the intersected modules. Inactive modules or regions such as edge areas on the silicon sensors are excluded from the hole definition.
} 

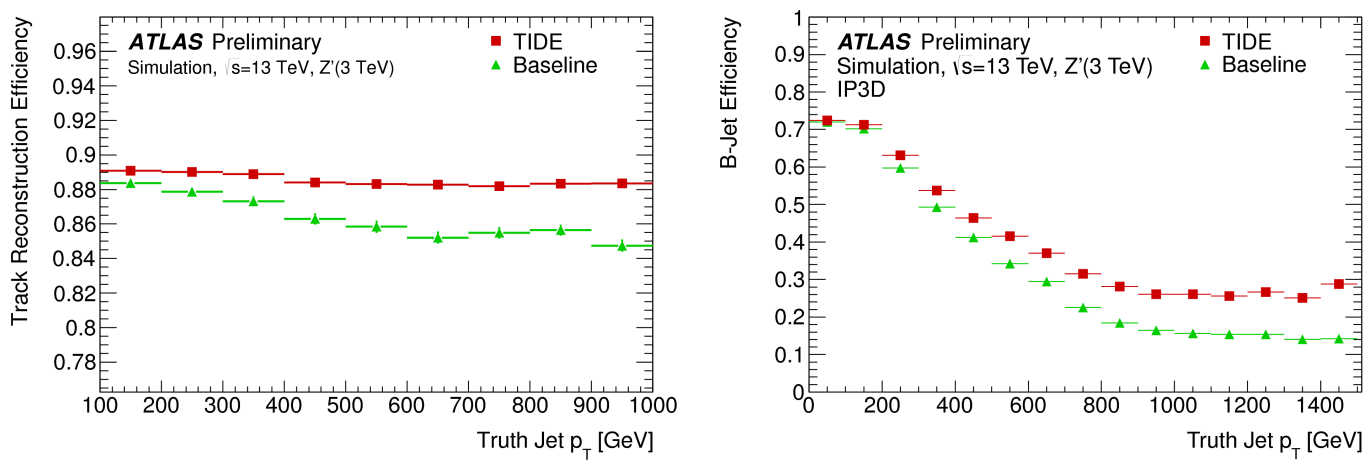

Figure 3: (Left) The average efficiency to reconstruct primary tracks with a production vertex before the first layer in jets as a function of jet $p_{\mathrm{T}}$. The same sample generation, with limited statistics, is used for both reconstruction algorithms resulting in correlated features. (Right) B-jet efficiency at the baseline $70 \%$ working point of the IP3D algorithm [9] as a function of truth-jet transverse momentum. The truth jets are reconstructed from generator-level particles in $Z^{\prime}$ events, using the anti- $k_{t}$ algorithm [10] with $R=0.4$, and are required to be within $|\eta|<2.5$. Two track reconstruction algorithms are shown: green triangles label the baseline reconstruction and red squares label the optimized reconstruction for dense environment. The Baseline legend represents the Run-1 algorithm, and the TIDE (Tracking In Dense Environment) legend represents the Run-2 algorithm [7].
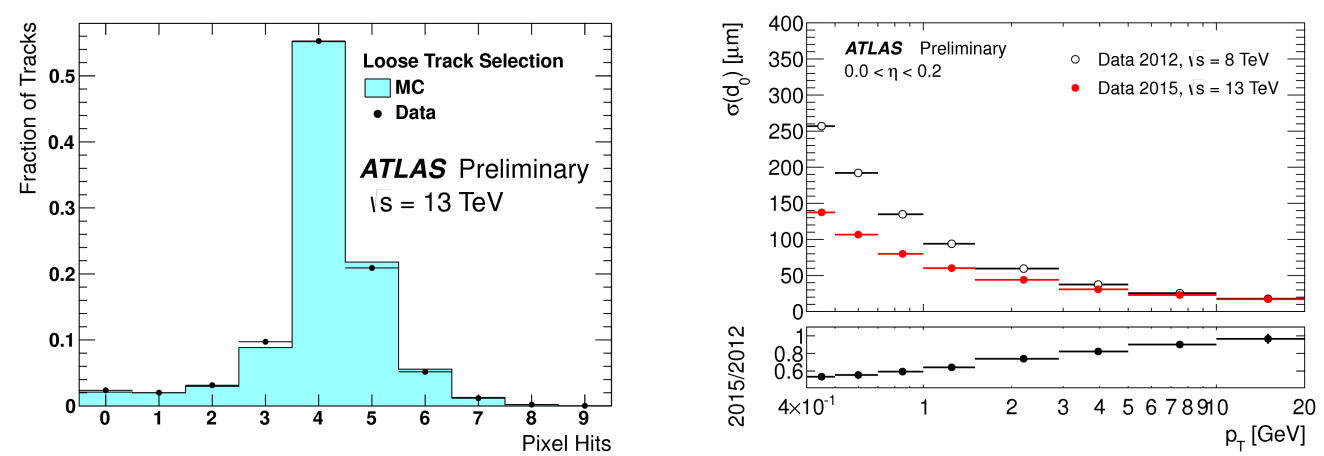

Figure 4: (Left) Comparison of the number of Pixel and IBL hits distributions in data and simulation (PYTHIA 8 A2:MSTW2008LO [13][14]). The distributions are normalized to one [11]. (Right) Unfolded transverse impact parameter resolution measured from data in $2015, \sqrt{s}=13 \mathrm{TeV}$, with the Inner Detector including the IBL, as a function of $p_{\mathrm{T}}$, for values of $0.0<\eta<0.2$ compared to that measured from data in 2012, $\sqrt{s}=8 \mathrm{TeV}$. The data in 2015 is collected with a minimum bias trigger. The data in 2012 is derived from a mixture of jet, $\tau$ and missing transverse energy triggers [12]. 

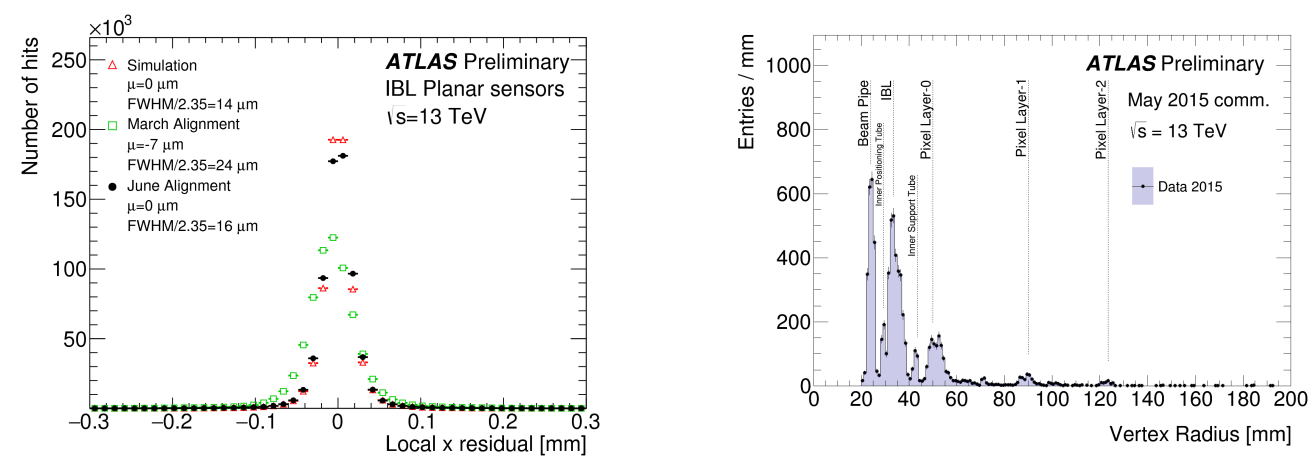

Figure 5: (Left) The local- $x$ residual distribution for the IBL planar sensors for the $13 \mathrm{TeV}$ collision data sample reconstructed with the June alignment (black) and March alignment (green) as well as observed in the perfectly aligned simulation (red). The distributions are integrated over all hits assigned to tracks in the respective IBL regions. The parameter $\mu$ represents the mean of the distributions. The distributions have been normalized to the same number of entries [16]. (Right) The radial vertex position distribution for hadronic interaction candidates reconstructed from multiple tracks with $>5 \sigma$ transverse impact parameter significance. These distributions depict structures consistent with hadronic interactions occurring within the new ATLAS beam pipe and IBL in addition to the original Pixel layers. The hadronic interaction candidates are required to be reconstructed within $r>20 \mathrm{~mm}$ and $|z|<300 \mathrm{~mm}$ [17].

parameter resolution in three dimensional way for the all data taking period. However, for the IBL a temperature-dependent distortion in $\phi$-direction was observed during commissioning using cosmic rays [15]. Therefore the possibility of the alignment correction, and potential impact of the misalignment due to instability of the distortion shape were decently studied. Figure 5 (Left) shows the hit residual distribution in the local- $x$ direction (corresponds to the $\phi$ direction in the ATLAS global coordinate system) after the alignment correction compared to the simulation of the nominal geometry [16]. In spite of the observed temperature-dependent shape change, the alignment is shown to correct for the distortion.

The material budget of the Inner Detector needs to be well described in the simulation to understand the tracking performance e.g. the track reconstruction efficiency. For Run-2 the material in the new components needs to be accurately assessed. Figure 5 (Right) shows the radial distribution of hadronic interaction vertex candidates using the Run-2 commissioning data, exhibiting peaks corresponding to materials of the IBL and Pixel detector layer structures [17].

\section{Summary}

The reconstruction of charged particle tracks in the ATLAS Inner Detector, in particular impact parameter resolution, was significantly improved in Run-2 compared to Run-1, both by software and hardware upgrades of the new IBL installation. The track reconstruction algorithm was overhauled, and the process is four times faster than the Run-1 reconstruction. Furthermore, reconsideration of the neural network consulting of the Pixel clusters made improvements of the tracking efficiency in dense environments. These improvements in tracking performance reported in this article provide a solid basis of the performance of the ATLAS experiment for versatile physics analyses using the Run-2 data at $\sqrt{s}=13 \mathrm{TeV}$. In this article, a number of early performance 
studies of the Run-2 Inner Detector and its track reconstruction is presented. The observed impact parameter resolution is significantly improved in the real collision data in Run-2 than in Run-1. Alignment corrections are performing well to give comparable residual distribution to the nominal simulation geometry. Evaluation of material budget and distribution using tracking techniques is also ongoing to determine the performance of the Inner Detector tracking.

\section{References}

[1] ATLAS Collaboration, The ATLAS Experiment at the CERN Large Hadron Collider, 2008 JINST 3 S08003.

[2] ATLAS Collaboration, ATLAS Insertable B-Layer Technical Design Report, CERN-LHCC-2010-013. ATLAS-TDR-19, Geneva, 2010

[3] Andreas Salzburger, on behalf of the ATLAS collaboration, Optimisation of the ATLAS Track Reconstruction Software for Run-2, ATL-COM-SOFT-2015-106, to be published in Journal of Physics: Conference Series (JPCS).

[4] http://proj-clhep.web.cern.ch/proj-clhep/

[5] http://eigen.tuxfamily.org/

[6] Eric Lançon on behalf of the ATLAS collaboration, Preparing ATLAS Distributed Computing for LHC Run 2, POS ( ISGC2014) 045

[7] ATLAS Collaboration, The Optimization of ATLAS Track Reconstruction in Dense Environments, ATL-PHYS-PUB-2015-006

[8] ATLAS Collaboration, A neural network clustering algorithm for the ATLAS silicon pixel detector, JINST 9 (2014) P09009, arXiv:1406.7690 [hep-ex]

[9] ATLAS Collaboration, Commissioning of the ATLAS high-performance b-tagging algorithms in the 7 TeV collision data, ATLAS-CONF-2011-102

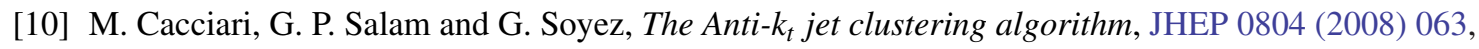
arXiv: 0802.1189 [hep-ph].

[11] ATLAS Collaboration, Track Reconstruction Performance of the ATLAS Inner Detector at $\sqrt{s}=13 \mathrm{TeV}$, ATL-PHYS-PUB-2015-018

[12] ATLAS Collaboration, Impact parameter resolution Run-1 vs. Run-2, IDTR-2015-007

[13] T. Sjostrand, S. Mrenna and P. Z. Skands, A Brief Introduction to PYTHIA 8.1, Comput.Phys.Commun. 178 (2008) 852-867, arXiv: 0710.3820 [hep-ph].

[14] A. Sherstnev and R. Thorne, Parton Distributions for LO Generators, Eur.Phys.J. C55 (2008) 553-575, arXiv: 0711.2473 [hep-ph].

[15] ATLAS Collaboration, Study of the mechanical stability of the ATLAS Insertable B-Layer, ATL-INDET-PUB-2015-001

[16] ATLAS Collaboration, Alignment of the ATLAS Inner Detector with the initial LHC data at $13 \mathrm{TeV}$, ATL-PHYS-PUB-2015-031

[17] ATLAS Collaboration, Hadronic Interaction Candidates in 13 TeV data, IDTR-2015-003 